\title{
Prolonged, but not complicated, grief is a mental disorder
}

\author{
Paul K. Maciejewski and Holly G. Prigerson
}

\section{Summary}

The DSM and ICD have taken steps to introduce a grief disorder as a new diagnostic entity. Evidence justifies the inclusion of prolonged grief disorder, but not complicated grief, as a new mental disorder.

\section{Declaration of interest}

The authors have no conflicts of interest.

\section{Copyright and usage}

(C) The Royal College of Psychiatrists 2017
Paul K. Maciejewski (pictured) is an Associate Professor of Biostatistics in Radiology, and Co-Director, Cornell Center for Research on End-of-Life Care at Weill Cornell Medicine. His research focuses on psychological, social and cultural influences on end-of-life medical decision-making and care, and surviving caregiver bereavement adjustment. Holly G. Prigerson is the Irving Sherwood Wright Professor in Geriatrics, Professor of Sociology in Medicine, and Co-Director, Cornell Center for Research on End-of-Life Care, at Weill Cornell Medicine. Her research focuses on psychosocial influences on end-of-life care and surviving caregiver bereavement adjustment.

\section{Introduction}

Leading authorities in psychiatry, and medicine more generally, now agree that available evidence justifies the inclusion of a grief disorder in the DSM and ICD. Despite agreement that prolonged, intense, disabling grief constitutes a mental disorder, there is a lack of agreement on the name of the disorder and on the criteria that clinicians should use to assess it. The new disorder, and comprehensive evidence in support of it, were originally introduced in a proposal for prolonged grief disorder (PGD). Following this proposal for PGD and its presentation to the DSM-5 subwork group, an alternative proposal for complicated grief appeared in a review article. ${ }^{2}$ The proposal for complicated grief was absent of any evidence that it accurately diagnosed the underlying grief disorder. Resolution of the conflict between these two proposals will remove any obstacles to consensus on criteria that has impeded progress in diagnosis and treatment of the many individuals who suffer from pathological grief. Here we provide a rationale and evidence in support of PGD, as opposed to complicated grief, as the criterion standard for disordered grief.

\section{Background}

In 1999, we published a preliminary report in the BJPsych ${ }^{3}$ that tested consensus criteria for disordered grief. A National Institutes for Health/National Institute of Mental Health-funded field trial, the Yale Bereavement Study (YBS), then followed to refine and test those criteria. That study produced a proposal and diagnostic criteria for PGD, ${ }^{1}$ defined as a genuine disorder of grief, characterised by intense, unrelenting grief-specific symptoms of loss such as yearning and physical or emotional suffering caused by the wanted, but unfulfilled, reunion with the deceased. This empirical study, published in $2009,{ }^{1}$ demonstrated that PGD met well-established criteria required for the validation of a new mental disorder. ${ }^{4}$
Following the 2009 publication, which demonstrated the validity of the PGD proposal, ${ }^{1}$ we made our case for PGD as a new diagnostic category directly to members of the DSM and ICD work groups. Both took the proposal for PGD seriously and have taken steps to introduce it into their diagnostic classification systems. The DSM expanded criteria for PGD to include unexamined elements of complicated grief, and renamed the disorder persistent complex bereavement disorder (PCBD). The ICD simplified the validated PGD criteria by reducing the number of symptoms to be considered for a diagnosis but retained its name, PGD. A recent analysis comparing these criteria demonstrated that PGD criteria, the DSM's more elaborate PCBD criteria and the ICD's simpler PGD criteria, but not complicated grief criteria, are all substantively the same and have strong diagnostic properties. ${ }^{5}$

\section{Evaluation of the evidence regarding complicated grief as a mental disorder}

To date, there is no evidence that complicated grief criteria, despite its obvious overlap and similarities with PGD, accurately identify individuals with a distinct mental disorder. Recently, we found that complicated grief had moderate agreement with PGD and PCBD. We identified $30 \%$ of our YBS community sample as having complicated grief as opposed to $10-15 \%$ for PGD and/or PCBD, that it produced more false positives (63\%) than true positives $(37 \%)$ with respect to our criterion standard, and, unlike PGD and PCBD, complicated grief had no predictive validity with respect to future mental disorder, functional impairment or diminished quality of life. ${ }^{5}$ Thus, complicated grief proved to be the unsubstantiated proposal in that it, unlike the other formulations, it did not meet accepted criteria ${ }^{4}$ for defining a mental disorder.

The assertion that complicated grief is not a mental disorder is at odds with the main conclusion of another recent report. Cozza et $a l^{6}$ compared PCBD, PGD and complicated grief criteria and concluded that the complicated grief criteria are superior to those for PCBD and PGD. We disagree with this conclusion, and given rates of overdiagnosis and flaws with the study design employed by Cozza et al, we draw the opposite conclusion. Concerns with the Cozza et al methodology and conclusions are detailed below.

The complicated grief criteria are, in our opinion, too easily satisfied. Not only is the number of symptoms to meet the complicated grief criteria too few - just one 'category B' and two 'category C' symptoms - but also the threshold required in Cozza et al for each symptom is too low - only 'moderate' (severity 
$\geqslant 3$ on a five-point Likert scale). This would mean, for example, that a bereaved individual with moderate yearning for the deceased and two additional symptoms such as moderate 'reluctance to pursue interests since the loss ...' and moderate 'difficulty with positive reminiscing about the deceased' would meet complicated grief criteria for pathological grief. As mentioned above, in a recent World Psychiatry report, ${ }^{5}$ we found that the positive complicated grief test rate in our YBS community sample was 30\% when we apply our standard symptom threshold of 'quite a bit' (severity $\geqslant 4$ on a five-point Likert scale) for each symptom. If we use Cozza et al's 'moderate' symptom threshold, then the positive complicated grief test rate in our YBS sample is $62 \%{ }^{7}$ A diagnostic test that identifies a majority of individuals in an ordinary, community-based, bereaved sample as having pathological grief contradicts the notion that most grief reactions are normal.

Figure 1 illustrates the relationships between all grief, complicated grief and PGD in the YBS sample based on Cozza et als 'moderate' symptom threshold for diagnosis of complicated grief and our conventional 'quite a bit' symptom threshold for PGD. Bereavement is a common, ubiquitous life event. Institutionalisation of complicated grief criteria in the DSM would translate into many normal bereavement reactions being diagnosed as a form of mental illness. From a public health perspective, criteria that diagnose most reactions to a natural life event as a psychiatric disorder lack credibility.

The fatal flaw of Cozza et al's study disqualifying it from serious consideration can be summarised in two words: 'spectrum bias'. Cozza et al removed nearly half $(n=797,46 \%)$ of their total sample from their main analysis to focus only on extreme 'cases' ( $n=260,15 \%$ of total sample) and 'controls' ( $n=675,39 \%$ of total sample). Long ago, Ransohoff \& Feinstein ${ }^{8}$ described the problem of 'spectrum bias' associated with study designs that exclude the most difficult cases to diagnose in favour of the most obvious cases and controls. Diagnosticians have cautioned against such case-control designs ${ }^{9}$ ever since because they overestimate the sensitivity and specificity of diagnostic tests by omitting diagnostic errors from near-threshold cases. The value of a diagnostic formulation's ability to discriminate between cases and controls is not in identifying extremes, but rather in discerning the cases that lie in between these extreme groups of obviously normal and obviously pathological cases. Undoubtedly, Cozza et al's spectrum-biased design conceals many false-positive diagnostic tests for complicated grief in the large segment (46\%) of their sample excluded from their main analysis. This critical information was neither reported in Cozza et al's study, nor provided to us by the authors after requests for these numbers (personal communication).

Beyond our fundamental concerns about the ease with which complicated grief criteria may be satisfied and Cozza et al's logic, design and analysis, there are some striking commonalities between their study and ours. Data from Cozza et $a l^{6}$ and our $\mathrm{YBS}^{5}$ are consistent with three basic facts. First, PGD and PCBD, and not complicated grief, represent the same diagnostic entity. Second, the rate of diagnosis of complicated grief is two to three times greater than that of PGD and/or PCBD. Third, individuals who meet criteria for PGD (and/or PCBD) are a subset of those who meet criteria for complicated grief. This means that complicated grief (using a symptom severity threshold of $\geqslant 4$ on a five-point Likert scale) is composed of a substantial minority of individuals $(\sim 40 \%)$ who have PGD, i.e. a genuine disorder of grief, and a majority of individuals $(\sim 60 \%)$ who do not. This $60 \%$ is a group we consider misclassified (i.e. wrongly pathologised) by the complicated grief criteria.

Thus, evidence from two independent, federally funded community-based bereavement studies, including Cozza et al's

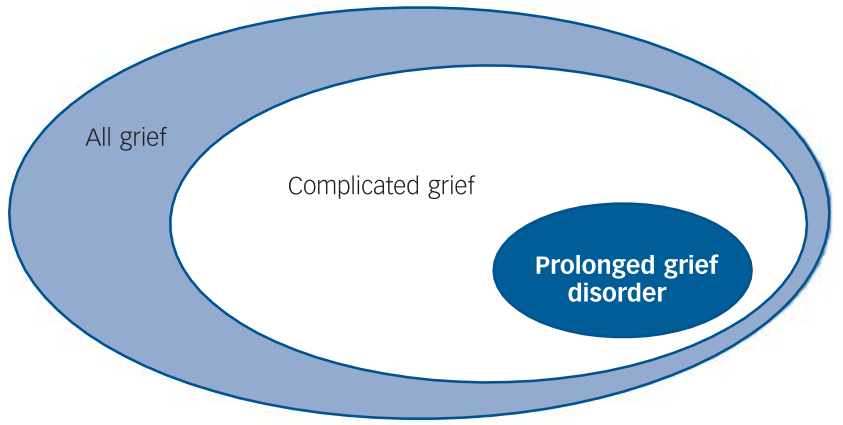

Fig. 1 Relationships between all grief, complicated grief and prolonged grief disorder in the Yale Bereavement Study (YBS) sample.

All grief, $100 \%$ of all bereaved individuals in the YBS sample; complicated grief identified using Cozza et al symptom threshold, $62 \%$ of all bereaved individuals in the YBS sample; prolonged grief disorder using Prigerson et al symptom threshold, $12 \%$ of all bereaved individuals in the YBS sample.

own data, lead us to reject Cozza et al's assertion of complicated grief criteria's superiority. Cozza et al conclude that the DSM should modify its PCBD criteria to be more in line with theirs. However, this would mean basing DSM criteria on complicated grief criteria that have not been empirically validated, that overdiagnose/pathologise normal grief and that produce more false than true positives. In fact, the evidence converges in support of unifying behind PGD as the diagnostic standard for disordered grief.

\section{Conclusions}

The purpose of diagnostic criteria is to provide a uniform and agreed upon (i.e. standardised) method for determining which individuals are truly suffering from a legitimate disorder. Valid and reliable diagnoses are a prerequisite for determining appropriate treatment; they should not, however, be used as a device for identifying whom to treat. PGD, and the DSM and ICD versions of it, accurately and reliably diagnose bereaved individuals who experience significant psychological distress and dysfunction, avoid pathologising normal bereavement reactions and identify individuals for whom severe and debilitating grief is likely to endure without effective intervention. We believe that PGD criteria achieve these goals and would be an empirically supported and clinically useful addition to the DSM and ICD.

Paul K. Maciejewski, PhD, Department of Radiology, Weill Cornell Medicine, New York, USA; Holly G. Prigerson, PhD, Department of Medicine, Weill Cornell Medicine, New York, USA

Correspondence: Paul K. Maciejewski, Weill Cornell Medicine, Department of Radiology, 525 East 68th Street, Box 141, New York, NY 10065, USA. Email: pam2056@med.cornell.edu

First received 2 Jan 2017, final revision 17 Apr 2017, accepted 10 May 2017

\section{References}

1 Prigerson HG, Horowitz MJ, Jacobs SC, Parkes CM, Aslan M, Goodkin K, et al. Prolonged grief disorder: psychometric validation of criteria proposed for DSM-V and ICD-11. PLOS Med 2009; 6: e1000121.

2 Shear MK, Simon N, Wall M, Zisook S, Neimeyer R, Duanet $\mathrm{N}$, et al. Complicated grief and related bereavement issues for DSM-5. Depress Anxiety 2011; 28: 103-17. 
3 Prigerson HG, Shear MK, Jacobs SC, Reynolds CF 3rd, Maciejewski PK, Davidson JR, et al. Consensus criteria for traumatic grief. A preliminary empirical test. Br J Psychiatry 1999; 174: 67-73.

4 Feighner JP, Robins E, Guze SB, Woodruff RA, Winokur G, Munoz R. Diagnostic criteria for use in psychiatric research. Arch Gen Psychiatry 1972 26: $57-63$.

5 Maciejewski PK, Maercker A, Boelen PA, Prigerson HG. "Prolonged grief disorder" and "persistent complex bereavement disorder", but not "complicated grief", are one and the same diagnostic entity: an analysis of data from the Yale Bereavement Study. World Psychiatry 2016; 15: 266-75
6 Cozza SJ, Fisher JE, Mauro C, Zhou J, Ortiz CD, Skritskaya N, et al. Performance of DSM-5 persistent complex bereavement disorder criteria in a community sample of bereaved military family members. Am J Psychiatry 2016; 173: 919-29.

7 Prigerson HG, Maciejewski PK. Rebuilding consensus on valid criteria for disordered grief. JAMA Psychiatry 2017; 74: 435-6.

8 Ransohoff DF, Feinstein AR. Problems of spectrum and bias in evaluating the efficacy of diagnostic tests. N Engl J Med 1978; 299: 926-30.

9 Lijmer JG, Mol BW, Heisterkamp S, Bonsel GJ, Prins MH, van der Meulen JH, et al. Empirical evidence of design-related bias in studies of diagnostic tests. JAMA 1999; 282: 1061-6.

\section{psychiatry} in literature

\title{
The Hunger Games: a portrayal of PTSD in teenage fiction
}

\author{
Nishan Ghoshal, Paul O. Wilkinson
}

The Hunger Games is a young adult dystopian fiction trilogy written by Suzanne collins. The books have sold over 65 million copies in the USA alone and have been adapted into a hugely successful film series.

The series follows the story of Katniss Everdeen, a 16-year-old girl living under an oppressive government that forces her to fight to the death against 23 other children in an annual event known as the Hunger Games. Katniss is made to go through the Games twice, during which she experiences numerous life-threatening events, including being chased by a wall of fire, blistered by toxic gas and hunted by packs of mutated animals. During the Games, she is forced to kill other children who are competing against her and suffers the loss of friends, one of whom dies in her arms. All this is amidst a constant battle to procure food and water, and indeed she nearly dies of thirst in one of the Games.

While she manages to escape both Games with her life, she is left suffering significant psychological distress and displays symptoms consistent with post-traumatic stress disorder (PTSD). She suffers from vivid flashbacks persisting for much more than a month after the Games; in one she envisions herself back in the arena, holding the bloodied body of her dying friend. Sleep difficulties and nightmares are also a recurrent problem and she is described as screaming and flailing about in her sleep. Her nightmares often seem to stem from feelings of survivor's guilt, for example dreaming that she is being buried alive by the loved ones for whose deaths she felt responsible.

She is hypervigilant long after imminent threat has passed, displaying exaggerated reactions to innocuous stimuli; in one case, the smell of roses (a reminder of the Games) causes her to dive behind a curtain in fear of imminent attack. Physical symptoms such as headaches and nausea are also a frequent complaint. She displays avoidance behaviour, including avoiding her friend who participated in the Games with her, as well as aversion to any form of discussion of the Games

Prior to the Hunger Games, Katniss experiences several predisposing factors for PTSD. The death of her father appears to cause severe depression in her mother. This leaves 11-year-old Katniss as the sole carer of her mother and younger sister. The resultant poverty leads to frequent hunger and Katniss having to hunt illegally to feed the family. By assuming a young carer role, Katniss is forced to mature over-quickly and has little time to socialise, leading to a lack of friends. Her mother's depression increases Katniss' genetic risk for PTSD, given common genetic risks for both disorders.

All this is set against a backdrop of an oppressive government. Katniss is constantly looking over her shoulder in fear that her illicit hunting activities will get her and her family executed. This background of constant threat serves as a significant psychological predisposing factor to PTSD.

The Hunger Games provides a powerful portrayal of PTSD, detailing clear symptoms of re-experiencing, hypervigilance and avoidance after life-threatening events, and presenting them in a manner that is accessible to its largely teenage readership. By employing detailed first-person emotional accounts set against a bleak dystopian narrative, it gives young readers the opportunity to explore how significant trauma can leave psychological scars long after the physical ones have healed. 